Some of our best results following the use of radium in the recurrent group have been in the infraclavicular region. In some of these cases, applications of a radium pack have resulted in a complete disappearance of the growth. One of the patients has now gone one and a half years with no further evidence of disease to be made out. The details of radium therapy and the methods employed have been fully described in a recent article, ${ }^{1}$ and will not be described here.

\section{TYPES BEST TREATED BY THE ROENTGEN RAY}

Recurrences best treated by the roentgen ray include diffuse cutaneous involvement, examples of so-called inflammatory carcinoma, extensive involvement of nodes in the axillary or supraclavicular regions, and mediastinal or pleural metastases, as well as bony metastases, in various parts of the body. The technic employed has been fully described in a recent paper. At present we are still depending mainly on the old type of roentgen-ray machine, and have been reasonably satisfied with the results obtained. Some of the most striking effects have been seen in the treatment of diffuse cutaneous involvement, an area of considerable disease disappearing in a few weeks under appropriate irradiation, leaving an apparently normal skin. In some of these cases, a later examination showed a reappearance of the lesion.

The new 200,000 volt machine now in operation is being used on carefully chosen patients, but sufficient time has not elapsed to make any report on this method of therapy. We are very hopeful that some of the mediastinal and pleural extensions of the disease may yield some encouraging results by this new method of treatment.

The gross and microscopic effects of the treatment of cancerous diseases by radium and the roentgen ray have been described elsewhere.

\section{CONCLUSIONS}

1. As a prophylaxis against the recurrence of breast carcinoma, a careful selection of patients for operation must be made.

2. Preoperative and postoperative cycles of roentgen ray are important prophylactic measures against recurrence.

3. A follow-up in every patient with carcinoma of the breast operated on should be adopted as a routine. No surgeon should operate on a patient with breast cancer without appreciating that an integral part of the procedure is following up the patient, with the idea in view of cliscovering the appearance of metastases at an early date, permitting the application of irradiation as soon as possible.

4. Properly applied irradiation to recurrent breast carcinoma definitely prolongs the life of the patient.

1 Lee, B. J.: Results to Date in the Treatment of Primary Inoperable Carcinoma of the Breast by Radiation, Ann. Surg., September, 1922.
5. I believe that ultimately, with more complete knowledge and better technic, a still further control of the recurrent phase of this disease may be expected.

128 East Seventy-Third Street.

\section{FOCAL INFECTION IN THE TONSIL CAUSING $A$ TUBTERCULOUS \\ OPHTHALMIA * \\ CECIL M. JACK, M.D. \\ DECATUR, ILL.}

A study of the etiology of chronic inflammatory conditions of the eye is instructive to both the internist and the ophthalmologist. We are beginning to believe that these conditions are not primary, but secondary to diseased foci in other parts of the body. It becomes

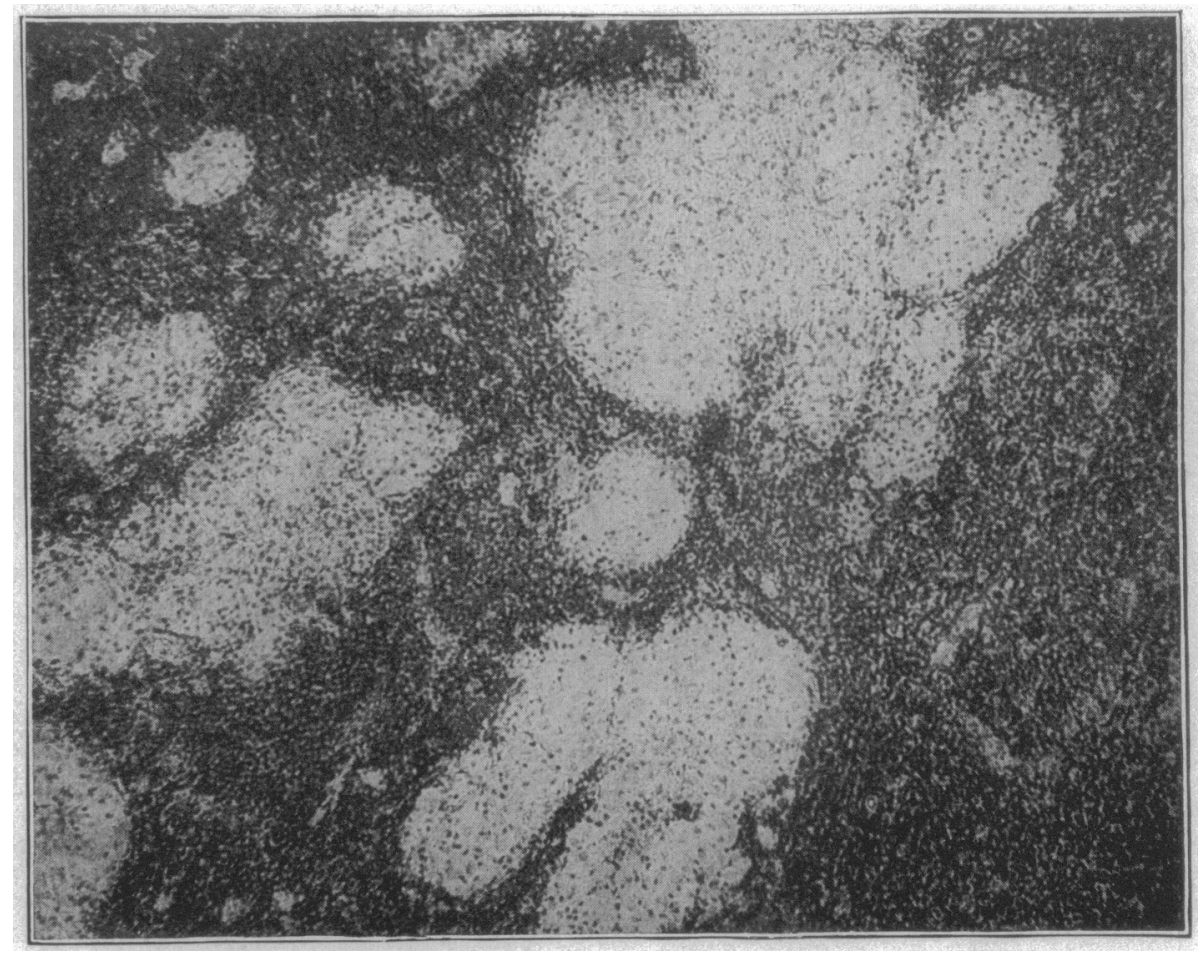
Fig. 1.- Section through tonsil: The smaller light areas are the individual epithelioid tubercles;
the larger confluent masses are the caseating ones; these are taken from around the infected crypt. the problem of the internist to search out these primary foci. The ophthalmologist has recognized tuberculous ophthalmia for some time, but has been content with such a diagnosis and treatment directed locally, or tuberculin given hypodermically. Those who considered it a secondary condition considered the lungs as a primary source and sought no further. The case I am reporting deals with such a condition. The primary focus was suggested by the history, but determined by a pathologic examination of diseased tissue removed.

\title{
REPORT OF CASE
}

History.-Miss B., aged 21, referred by Dr. A. E. Prince of Springfield, Ill., for examination and treatment, a diagnosis of tuberculous sclerokeratitis having been made, complained chiefly of an inflamed right eye with failing vision. The father died of diabetes at 72 ; the mother was living at 60 ; one brother was living and well. There had been no tuberculosis

* Read before the Decatur Medical Society, Dec. 20, 1921.

* Read before the Section on Ophthalmology at the Seventy. Third Annual Session of the American Medical Association, St. Louis, May,
1922. 
in the family. The patient had been delicate as a child, had been bottle fed, and at the age of 3 was operated on by Dr. John B. Murphy of Chicago for tuberculons cervical adenitis. She had many attacks of tonsillitis when older, and a tonsil operation was performed at the age of 14 , since which time she has been free from sore throat. At 18, March 16, 1916, she began having an inflammation of the right eye. She consulted several physicians, Dr. Prince being the first (June, 1916) to make the diagnosis of tuberculous ophthaimia. The first attack lasted six months, and was treated by Dr. George Palmer of Springfield with tuberculin. She remained well until March, 1920, when the inflammation returned. She was in Chicago at this time, and consulted $\mathrm{Dr}$. William $\mathrm{E}$. Gamble, who agreed in the diagnosis. His notes show that the vision was normal, and that there was a mild ciliary injection and a cloudy iris, which did not react well to light. The patient then moved to Decatur, and at this time came under my observation. Tuberculin was given, and she seemed to improve at first; but the process took a turn for the worse, the vision became impaired, and the other eye became inflamed. Tuberculin was stopped, and the eyes were put at rest with atropin. The condition continued to grow worse. She now consulted Dr. William H. Wilder of Chicago, who agreed that the process was tuber. culous, and it was his opinion that there was some question as to whether the opacities of the cornea would allow her to have even useful vision in the right eye. The vision at this time was $1 / 200$.

Physical Examination.-There was marked conjunctivitis of the right eye, a diffuse opacity of the cornea, greater in the lower part, and a marked scleritis, together with uveitis. The vision of the eye was $1 / 200$.

There was considerable conjunctivitis and ciliary injection of the left eye. The cornea and the interior of the eye showed no evidence of any lesion when viewed with the ophthalmoscope; the vision was $20 / 30$.

Between the tonsillar pillars, stumps of tonsil tissue could be seen, especially on the right side. A scar showed on the neck where the tuberculous glands had been removed at 3 years of age. The rest of the physical examination revealed nothing abnormal. Complete chest and gastrointestinal examination was made, including the use of the roentgen ray. This examination was made in January, 1921. The outlook was so unfavorable at this time that I had decided to make a complete reexamination. In taking the history, the patient's mother told me with what difficulty the tonsils were removed - that she had to go back a number of times for treatment and the removal of small pieces. This history and the scar on the neck led me to consider the remaining stumps of tonsil tissue as a focus, and I advised their enucleation.

Operation and Result.-At the time of the operation (February, 1921) the vision was so impaired that the patient could not recognize her mother in the same room. The operation was performed under local anesthesia. A piece, the size of a hazelnut, was enucleated from the right side, and from the left three pieces which were completely covered by a slitlike fold were recovered, Dr. A. S. Warthin of Ann Arbor, Mich., made the pathologic report: The tonsil showed a condition of chronic inflammation and hyperkeratosis. It contained great numbers of miliary tubercles clustered around the crypts. The majority of these were epithelioid tubercles, but the larger confluent ones showed caseation and giant cells. No tubercles were found in the germ centers. so that the process was a crypt infection, and not a hematogenous one. One crypt was completely surrounded by a confluent mass of miliary tubercles. From this report one might think that there were two processes at work: an older one as shown by the caseation, the other a more recent one, shown by the miliary tubercles.

Improvement took place rapidly, and now, nine months after the operation, her vision in the right eye has returned to $20 / 50$. and in the left eye to $20 / 20$. The opacities in the right eye have cleared beyond all expectation. The left is a perfect eye.

\section{COMMENT}

In searching for similar cases, advantage was taken of the American Institute of Medicine of New York. They made for me a diligent search, but the information obtained was very meager. No case of tuberculous sclerokeratitis could be found in which a pathologic report revealed the presence of tuberculosis in the tonsil.

I record this case, because in searching through the literature no like observation could be found, and I hope that this report will stimulate others to make

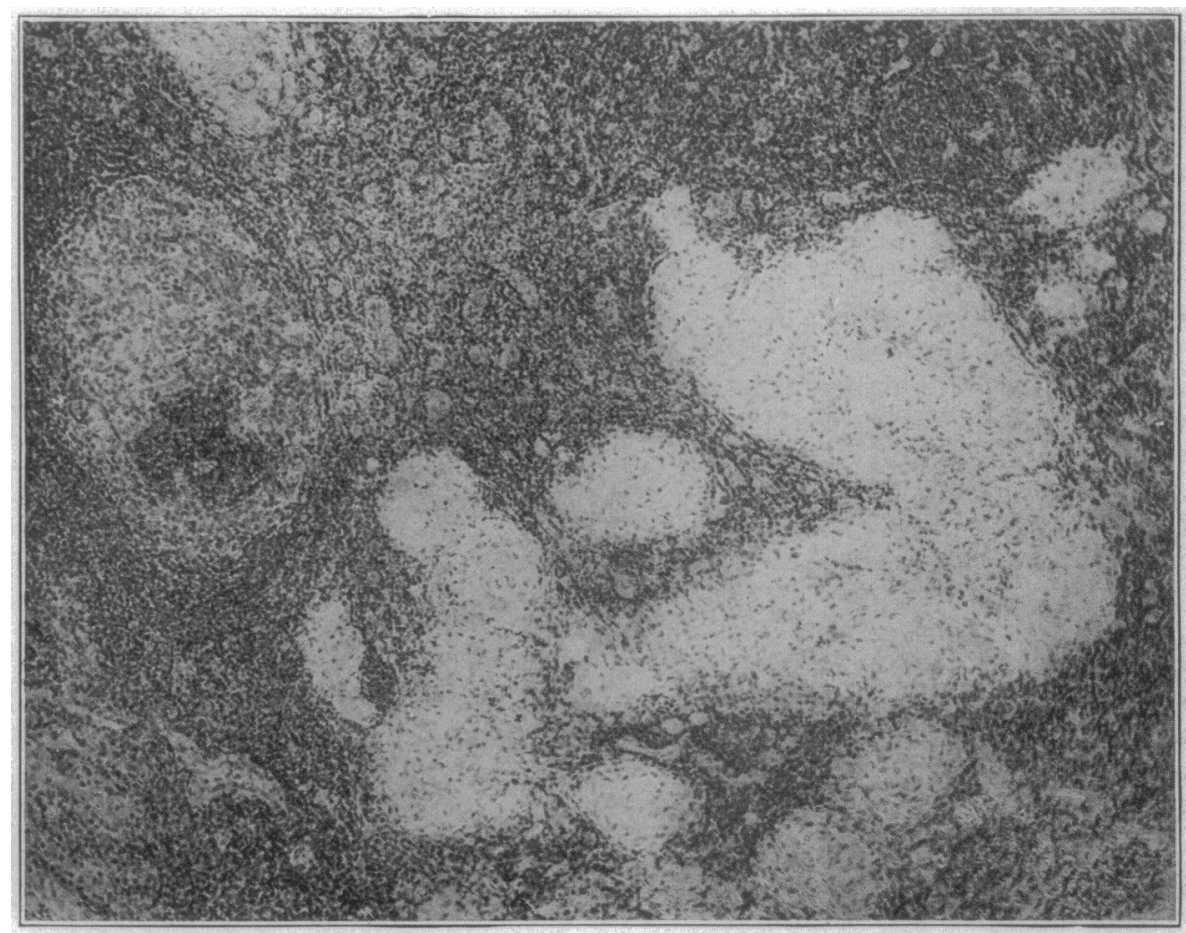

Fig. 2.-Section through tonsil: germ center on left, which is free from tubercle.

pathologic examination of the tissue removed when a like condition appears.

134 West Prairie Avenue.

\section{ABSTRACT OF DISCUSSION}

Dr. Oliver Tydings, Chicago: Many years ago a patient consulted me and I sent her to Dr. Baidwin of Columbus, Ohio, for operation. He operated and sent me the pathologic report. It was a uterine disturbance, a tuberculous condition. Fifteen years afterward that patient returned with a tuberculous condition of the retina. She was given tuberculin. She has recovered perfectly normal vision. I have seen her within the last six months and her eyes are normal.

Dr. William C. FinnofF, Denver: I do not recall ever seeing the tonsil referred to as the primary focus, although there is no reason why it should not be. I believe that in many cases of chronic ocular tuberculosis the primary focus is in the lymph glands, and probahly in some cases the primary focus is in the tonsil. Some years ago I examined 200 pairs of tonsils, and $I$ found tuberculosis in four of them, or 2 per cent. The first statement in the abstract, that tuber- 
culous ophthalmia is secondary to primary tuberculosis elsewhere, I think is a misstatement, because sclerokeratitis might be a primary condition. Intra-ocular tuberculosis in all instances, unless connected with injury, is secondary. The use of tuberculin in the treatment of a tuberculous eye condition is not specific. Hygienic treatment must be used as well. Tuberculin is just one therapeutic adjunct.

\title{
IMPROVEMENTS IN PREOPERATIVE, AND POSTOPERATIVE CARE*
}

\author{
F. B. TAYLOR, M.D. \\ W. I. TERRY, M.D. \\ AND \\ W. C. ALVAREZ, M.D. \\ SAN FRANCISCO
}

Until 1919, it was customary at the University of California Hospital to give a dose of castor oil the night before operation, and enemas in the morning. At that time, Dr. Terry discontinued the routine purgation, but left the order for enemas. The general impression about the hospital since then has been that the postoperative recoveries are on the average more satisfactory, and that there is less vomiting, abdominal distention and pain. Several of the operators are enthusiastic over the change, and no one has expressed any desire to return to the old routine. In 1918, Alvarez ${ }^{1}$ suggested that it would be wise also to abolish the routine purge usually given on the second or

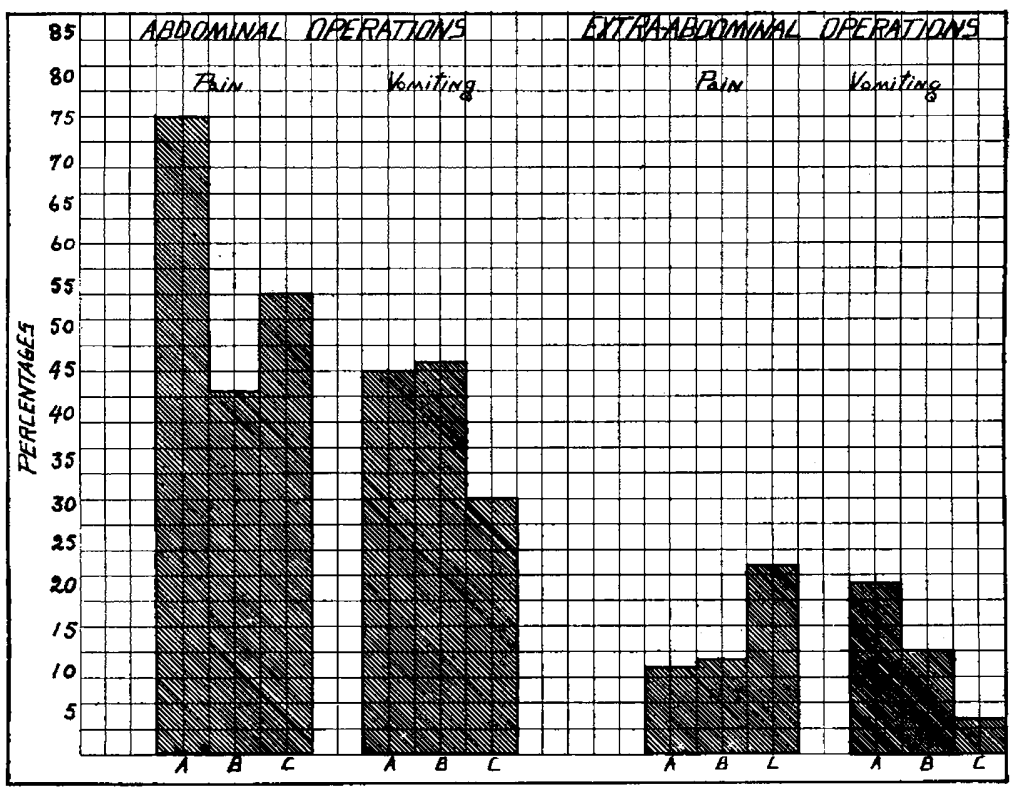

Incidence of pain and vomiting in patients: $A$, purged before and soon after operation; $B$, not purged before, but purged soon after operation; $C$, not purged before and not purged for four days after operation. undoubtedly be better sometimes if the operator would restrain himself a bit and wait patiently for Nature.

As some of our surgeons felt a certain amount of uneasiness about giving up such a long-established custom, it was thought wise to observe the results of the new technic very carefully and to compare them statistically with those obtained in the preceding period when patients were purged before and after operation.

An effort was made to answer the following two questions: (1) Has it been advantageous to give up the routine preoperative purgation ; and (2) has it been advantageous to give up the routine postoperative purgation?

Sixty-two of the people whose postoperative records have been analyzed in this paper were watched and questioned day by day by one of us (F. B. T.). Unfortunately, our conclusions in regard to the control group operated on before 1919 must be based solely on the nurses' records. Most of these are from the University of California Hospital; some are from Lane Hospital, some from the Children's Hospital and some from Mount Zion Hospital. Unfortunately, nurses are often kept pretty busy, and we cannot be sure that every complaint of pain and every attack of vomiting has been charted. However, on comparing the nurses' records in the last sixty-two cases with the notes made independently by Dr. Taylor, we feel that the nurses can be trusted to give a fair idea of the storminess or mildness of a postoperative period. One point in favor of the hospital records is that they were made without bias, i. e., the nurses had no idea of the use to which their notes were to be put. In anaiyzing these data, no attention has been paid to the patients' symptoms on the day of operation because we must expect some suffering at that time from the effects of the anesthetic and of the incision.

Our conclusions are based on records from 211 patients. Of these, 146 had abdominal, and sixty-five had extra-abdominal operations. The abdominal series includes appendectomies, hernioplasties, gastroenterostomies, cholecystectomies and explorations. As it would obviously not be fair to compare the postoperative symptoms after partial gastrectomies with those after simple hernioplasties, an effort has been made to have the different types of operations represented about equally in the control and the experimental groups. Aside from this, the cases were taken serially, without any selection or discrimination. The extra-abdominal group includes most of the common types of operation performed in a large hospital. 